\title{
Charge density wave and lock-in transitions of $\mathrm{CuV}_{2} \mathrm{~S}_{4}$
}

\author{
Sitaram Ramakrishnan, ${ }^{1}$ Andreas Schönleber ${ }^{1}$ Christian B. Hübschle,${ }^{1}$ Claudio Eisele,${ }^{1}$ Achim M. Schaller,${ }^{1}$ Toms Rekis, \\ Nguyen Hai An Bui, ${ }^{1, *}$ Florian Feulner, ${ }^{1,}$ Sander van Smaalen, ${ }^{1, \dagger}$ Biplab Bag, ${ }^{2}$ Srinivasan Ramakrishnan, ${ }^{2, \S}$ \\ Martin Tolkiehn, ${ }^{3}$ and Carsten Paulmann ${ }^{4}$ \\ ${ }^{1}$ Laboratory of Crystallography, University of Bayreuth, 95447 Bayreuth, Germany \\ ${ }^{2}$ Department of Condensed Matter Physics and Materials Science, Tata Institute of Fundamental Research, Mumbai 400005, India \\ ${ }^{3}$ P24, PETRA III, DESY, 22607 Hamburg, Germany \\ ${ }^{4}$ Mineralogisch-Petrographisches Institut, Universität Hamburg, 20146 Hamburg, Germany
}

(Received 21 March 2019; revised manuscript received 25 April 2019; published 23 May 2019)

\begin{abstract}
The three-dimensional charge density wave (CDW) compound $\mathrm{CuV}_{2} \mathrm{~S}_{4}$ is known to undergo phase transitions at $\sim 91$ and $\sim 50 \mathrm{~K}$. Employing single-crystal $\mathrm{x}$-ray diffraction on an annealed crystal, we confirm the formation of an incommensurate $\mathrm{CDW}$ at $T_{\mathrm{CDW}} \approx 91 \mathrm{~K}$, and we establish the nature of the transition at $T_{\text {lock-in }} \approx 50 \mathrm{~K}$ as a lock-in transition toward a threefold superstructure. As-grown crystals develop the same incommensurate CDW as the annealed crystal does, but they fail to go through the lock-in transition. Instead, the length of the modulation wave vector continues to decrease down to low temperatures in as-grown crystals. These findings are corroborated by distinct temperature dependencies of the electrical resistivity, magnetic susceptibility, and specific heat measured on as-grown and annealed crystals. A superspace model for the crystal structure of the incommensurate CDW suggests that the formation of extended vanadium clusters is at the origin of the CDW. In the lock-in phase, short and long V-V distances persist, but clusters now percolate the entire crystal. The lowering toward orthorhombic symmetry appears to be responsible for the precise pattern of short and long $\mathrm{V}-\mathrm{V}$ distances. However, the orthorhombic lattice distortion is nearly zero for the annealed crystal, while it is visible for the as-grown material, again suggesting the role of lattice defects in the latter.
\end{abstract}

DOI: 10.1103/PhysRevB.99.195140

\section{INTRODUCTION}

Historically, charge density waves (CDWs) have been associated with instabilities of quasi-one-dimensional (1D) metals $[1,2]$. The latter compounds feature parallel chains of atoms or molecules in their crystal structures, while the electrical conductivity is much higher along this unique direction than perpendicular to it. The CDW is stabilized by Fermi surface nesting (FSN) between coplanar sections of the Fermi surface. The wave vector $\mathbf{q}$ responsible for the FSN characterizes a wavelike variation of the valence electron density as well as a periodic lattice distortion (PLD) of the atoms. Depending on the filling of the valence band, $\mathbf{q}$ may be commensurate or incommensurate with the periodicities of the underlying lattice. Ideally, FSN involves the entire Fermi surface, and the phase transition between the normal state and the CDW state is a metal-insulator transition, as has been observed for blue bronze, $\mathrm{K}_{0.3} \mathrm{MoO}_{3}$ [3]. In most compounds, only a finite fraction of the Fermi surface is nested, and the CDW transition causes an anomalous increase of the electrical resistivity upon cooling through the transition, as has been found in $\mathrm{NbSe}_{3}$ [4].

\footnotetext{
*Present address: P3 Systems GmbH, 85055 Ingolstadt, Germany.

${ }^{\dagger}$ Present address: Instrument Systems Optische Messtechnik $\mathrm{GmbH}, 81677$ Munich, Germany.

${ }^{\ddagger}$ smash@uni-bayreuth.de

§ramky@tifr.res.in
}

FSN as the origin of the CDW transition has been criticized by Johannes and Mazin [5]. Instead, they proposed q-dependent electron-phonon coupling (EPC) to be the deciding interaction. It is now recognized that there can exist several disparate mechanisms for CDW formation, applicable in different compounds, which include FSN, EPC, orbital order, and charge ordering [6,7].

CDW instabilities have been recognized to develop in three-dimensional (3D) materials, which lack a clear anisotropy of their electrical conductivities and their crystal structures, but which involve strong electronic correlations [8]. Materials of this kind include $R_{5} \mathrm{Ir}_{4} \mathrm{Si}_{10}$, where $R$ is a rareearth element [9], $R \mathrm{NiC}_{2}$ [10-13], $R_{3} \mathrm{Co}_{4} \mathrm{Sn}_{13}$ [14], $\mathrm{Sr}_{3} \mathrm{Ir}_{4} \mathrm{Sn}_{13}$ [15,16], and $\mathrm{NiV}_{2} \mathrm{~S}_{4}$ [17]. Recent interest in CDW materials has been motivated by the interplay between CDW, superconductivity, and magnetic order. The competition between CDWs and superconductivity was experimentally demonstrated through the observation of enhanced superconductivity upon suppression of the CDW, for example as achieved by pressure in $\mathrm{Lu}_{5} \mathrm{Ir}_{4} \mathrm{Si}_{10}$ [18] and by chemical doping in $\mathrm{Cu}_{x} \mathrm{TiSe}_{2}(x \approx 0.05)$ [19].

$\mathrm{CuV}_{2} \mathrm{~S}_{4}$ is a prototypic 3D material, since its spinel structure has cubic symmetry $F d \overline{3} m$ and thus lacks anisotropy of its electrical resistivity by principle. Yet, it shows CDW transitions at $\sim 91$ and $\sim 50 \mathrm{~K}$. Furthermore, the low-temperature behavior of the specific heat and the magnetic susceptibility have suggested the presence of strong electron correlations [20,21]. This makes $\mathrm{CuV}_{2} \mathrm{~S}_{4}$ unique among the CDW materials. However, unlike other CDW materials, there is 
conflicting information on the low-temperature phase diagram. The initial report by Fleming et al. [22] proposed a CDW phase transition at $T_{\mathrm{CDW}}=90 \mathrm{~K}$ and a first-order phase transition at $T_{c 2}=50 \mathrm{~K}$ with a hysteresis of $\Delta T=10 \mathrm{~K}$. Single-crystal x-ray diffraction (SXRD) by Fleming et al. [22] indicated the development of an incommensurate CDW at $T_{\mathrm{CDW}}$ with modulation wave vector $\mathbf{q}=\left(\frac{3}{4}+\delta\right)(1,1,0)$ and a temperature-dependent incommensurability $\delta$ between 0.015 and 0 [23]. At $T=75 \mathrm{~K}$ the modulation became commensurate $(\delta=0)$, while the $50 \mathrm{~K}$ transition involved a change of $\mathbf{q}$ toward a different incommensurate value $\mathbf{q}^{\prime}=$ $\left(\frac{2}{3}+\delta^{\prime}\right)(1,1,0)$ with $\delta^{\prime} \approx 0.01[22]$.

Subsequent work confirmed two phase transitions, albeit at different $T_{\mathrm{CDW}}=91.5 \mathrm{~K}$ and $T_{c 2}=56 \mathrm{~K}$ with a hysteresis of only $4 \mathrm{~K}$ [24]. Specific-heat data resulted in $T_{\mathrm{CDW}}=92 \mathrm{~K}$ and $T_{c 2}=58 \mathrm{~K}$ [25]. Based on x-ray powder diffraction (XRPD), Okada et al. [24] discovered a tetragonal lattice distortion at all temperatures below $90 \mathrm{~K}$. More recently, again using XRPD, Kawaguchi et al. [26] determined the modulated structure of the incommensurate CDW at $70 \mathrm{~K}$, employing the orthorhombic superspace group $\operatorname{Imm} 2\left(0 \sigma_{o} 0\right)$ and modulation wave vector $\mathbf{q}=\left(\frac{3}{4}+\delta\right)(1,1,0)$ with $\delta=-0.0109$ (negative) [26]. An orthorhombic lattice distortion is in agreement with the number of six domains in the CDW phase as observed by electron diffraction [27]. However, Kawaguchi et al. [26] report the diffraction to be equal at 20 and $70 \mathrm{~K}$, thus failing to see any structural effects of the phase transition at $50 \mathrm{~K}$.

Horny et al. [21,28] have shown that the phase transitions of $\mathrm{CuV}_{2} \mathrm{~S}_{4}$ depend on the history of the sample, which they attributed to the presence of disorder in various degrees. Good-quality samples with a reproducible behavior can be obtained by annealing previously synthesized single crystals at $723 \mathrm{~K}$ for several days [21]. Here we report the results of SXRD experiments on as-grown and annealed $\mathrm{CuV}_{2} \mathrm{~S}_{4}$. For an annealed single crystal we find that the phase transition at $T_{\mathrm{CDW}}$ involves the development of a CDW with $\mathbf{q}=\left(\frac{3}{4}+\right.$ $\delta)(1,1,0)$ and a temperature-dependent incommensurability $\delta$. The $50 \mathrm{~K}$ transition is identified as a lock-in transition toward $\mathbf{q}_{c}=\frac{2}{3}(1,1,0)$. The modulated structure is refined against SXRD, employing the same orthorhombic superspace group as that used in [26]. However, modulation amplitudes are different for some atoms from their values obtained from Rietveld refinement against XRPD [26]. We can reproduce all the previously observed behavior in the present SXRD experiments on as-grown single crystals, including the absence of phase transitions in some crystals and different temperature dependencies of the modulation satellite reflections in other crystals. The present models for the CDW and lock-in phases are discussed in view of other highly correlated CDW materials and of the band structure of $\mathrm{CuV}_{2} \mathrm{~S}_{4}$.

\section{EXPERIMENT}

\section{A. Synthesis and x-ray diffraction}

Microcrystalline $\mathrm{CuV}_{2} \mathrm{~S}_{4}$ was prepared by direct reaction of the elements according to procedures discussed by Horny [21]. An evacuated and sealed quartz-glass ampoule containing a stoichiometric mixture of copper (381.2 mg; 99.999\% purity; Alfa Aesar), vanadium (635.8 mg; 99.5\%; Aldrich), and sulfur (769.6 mg; 99.998\%; Alfa Aesar), including 4\% excess of vanadium, was slowly heated to and then kept at $1153 \mathrm{~K}$ for $120 \mathrm{~h}$. Subsequently, the ampoule was cooled down to $293 \mathrm{~K}$ over $8 \mathrm{~h}$. Single crystals were grown by vapor transport in evacuated quartz-glass ampoules, employing $110 \mathrm{mg}$ of previously synthesized microcrystalline powder together with $5 \mathrm{mg} / \mathrm{cm}^{3}$ iodine as a transport agent [21]. After slow heating, the ampoule was kept for $96 \mathrm{~h}$ in a gradient of $1063 / 1003 \mathrm{~K}$, with the educt at the hotter side. Single crystals of $\mathrm{CuV}_{2} \mathrm{~S}_{4}$ grew in the colder half of the ampoule with sizes of up to $0.2 \mathrm{~mm}$. Different experiments led to crystals of different sizes, but never larger than $2 \mathrm{~mm}$. Attempts to grow single crystals in a gradient of 1093/1033 K-favorably discussed in the literature [21]—resulted in the formation of single crystals of almost exclusively $\mathrm{Cu}_{3} \mathrm{VS}_{4}$ [29]. Employing an exact stoichiometric mixture or an excess of sulfur also increases the amount of $\mathrm{Cu}_{3} \mathrm{VS}_{4}$ that is formed at the product side.

Crystals of suitable sizes for SXRD were taken from one batch. They were annealed for $48 \mathrm{~h}$ at $723 \mathrm{~K}$ in an evacuated quartz-glass ampoule [21]. One annealed crystal of good quality-crystal A-was used for the SXRD experiment at beamline P24 of the synchrotron PETRA III at DESY in Hamburg, Germany. SXRD was measured on a fourcircle diffractometer with a MARCCD detector, employing monochromatic radiation of wavelength $\lambda_{P 24}=0.495935 \AA$. The temperature of the sample was set at selected values between 20 and $250 \mathrm{~K}$ with a CRYOCOOL open-flow cryostat, employing helium as cryo gas.

As-grown single crystals from different batches were extensively tested by SXRD on a MAR345 image plate diffractometer with monochromatized $\operatorname{Ag} K \alpha$ radiation $(\lambda=$ $0.5608 \AA$ ) from a rotating anode source. Several crystals of suitable sizes (smaller than $0.2 \mathrm{~mm}$ ) were obtained, which were of excellent quality (all peaks indexed with the cubic lattice; narrow Bragg reflections). The quality of annealed and as-grown single crystals cannot be distinguished on the basis of these criteria.

Temperature-dependent SXRD was measured on several as-grown single crystals at the Swiss-Norwegian Beamlines (SNBL) of the European Synchrotron Radiation Facility (ESRF) in Grenoble [30]. A one-circle diffractometer was used together with a PILATUS $2 \mathrm{M}$ detector placed perpendicular to the beam, employing monochromatic radiation of wavelength $\lambda_{\mathrm{SNBL}}=0.67760 \AA$ A. In a first experiment, crystal $\mathrm{B}$ was cooled with a nitrogen open-flow cryostat toward temperatures between 80 and $300 \mathrm{~K}$. In particular, diffraction was measured at $1 \mathrm{~K}$ intervals between 80 and $95 \mathrm{~K}$, encompassing the CDW transition.

In a second experiment, an open-flow helium cryostat was used to cool the samples at temperatures between 4.2 and $45 \mathrm{~K}$ [31]. This setup was prone to the formation of ice around the samples. Ice formation prevented some datasets from being indexed, while reliable values for integrated intensities of Bragg reflections could not be obtained for several more datasets, even though reliable values for the lattice parameters and modulation wave vector were obtained. Useable data were thus obtained at six temperatures for crystal C, at eight temperatures for crystal D, and at a single temperature for crystal E. 


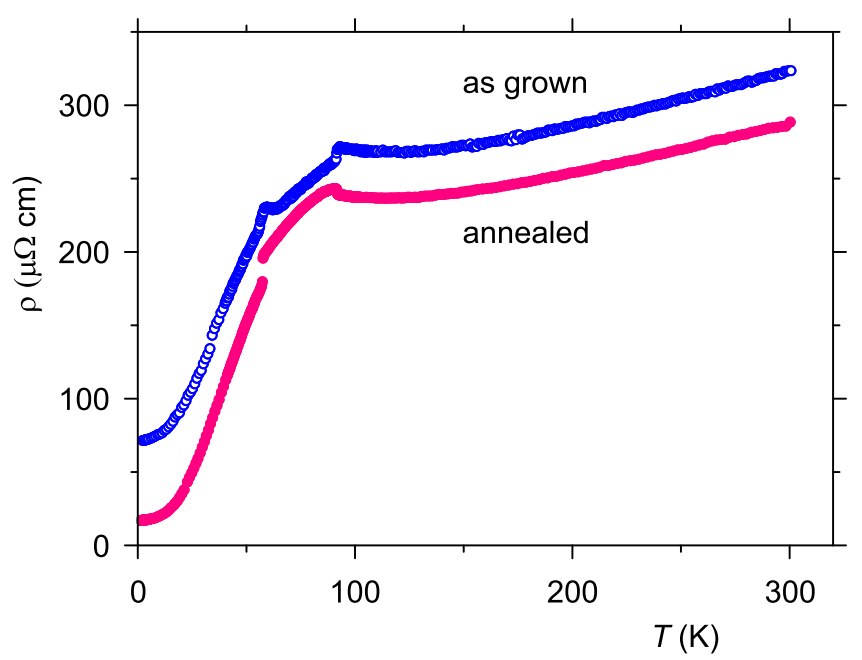

FIG. 1. Temperature dependence $(2-300 \mathrm{~K})$ of the electrical resistivity $(\rho)$ of $\mathrm{CuV}_{2} \mathrm{~S}_{4}$, as measured on an as-grown crystal (blue open circles) and on the same crystal after annealing (filled red dots).

\section{B. Physical properties}

Physical properties and their dependence on temperature have been measured on a large, bar-shaped single crystal of $\mathrm{CuV}_{2} \mathrm{~S}_{4}$ : crystal $\mathrm{P}$ from batch KK216. Properties were initially measured on the as-grown crystal P. Subsequently, crystal $\mathrm{P}$ was annealed for one week at $723 \mathrm{~K}$. Properties were again measured, then producing values for the annealed state.

The electrical resistivity was measured at temperatures from 2 to $300 \mathrm{~K}$, employing a laboratory-built setup at the Tata Institute of Fundamental Research (TIFR) in Mumbai. Ohmic contacts have been made using conductive silver paste and gold wires $(40 \mu \mathrm{m}$ diam) for the standard four-probe technique configuration to measure resistivity. Electrical resistivity was measured using a LR-700 (Linear Research, USA) ac bridge with $5 \mathrm{~mA}$ current operating at a frequency of $16 \mathrm{~Hz}$. The electrical resistivities of both the as-grown and annealed states of crystal $\mathrm{P}$ reveal anomalies at $\sim 91$ and $\sim 56$ K, i.e., at both transitions (Fig. 1). These anomalies are sharper and more pronounced in the annealed state, in agreement with better quality of the latter state. The resistivity is smaller for the annealed state by an amount of $35.1 \mu \Omega \mathrm{cm}$ at room temperature and by an amount of $54.6 \mu \Omega \mathrm{cm}$ at low temperatures. This indicates that annealing indeed removes much of the defects. The excellent quality of the crystal in the annealed state also shows up in the residual resistance ratio $\left(\mathrm{RRR} ; \rho_{300 \mathrm{~K}} / \rho_{4.2 \mathrm{~K}}\right.$ ) being equal to 16.5 , which is comparable to the best reported values [21], and being substantially larger than 4.5 for the as-grown crystal.

The heat capacity $\left(C_{p}\right)$ was measured by the thermal relaxation method using a Physical Property Measuring unit (PPMS, Quantum Design, USA). Data on the as-grown state were collected for 5-250 K. The annealed crystal $\mathrm{P}$ was measured for 5-150 K. $C_{p}(T)$ of the annealed crystal exhibits anomalies at $T_{\mathrm{CDW}}=90.37 \mathrm{~K}$ and $T_{c 2}=56.68 \mathrm{~K}$, whereas $C_{p}(T)$ of the as-grown state has an anomaly at $T_{\mathrm{CDW}}=$ $87.08 \mathrm{~K}$ only (Fig. 2). Clearly, the sharp feature at lower temperature is related to the lock-in transition as observed by the x-ray measurements. The low-temperature heat-capacity

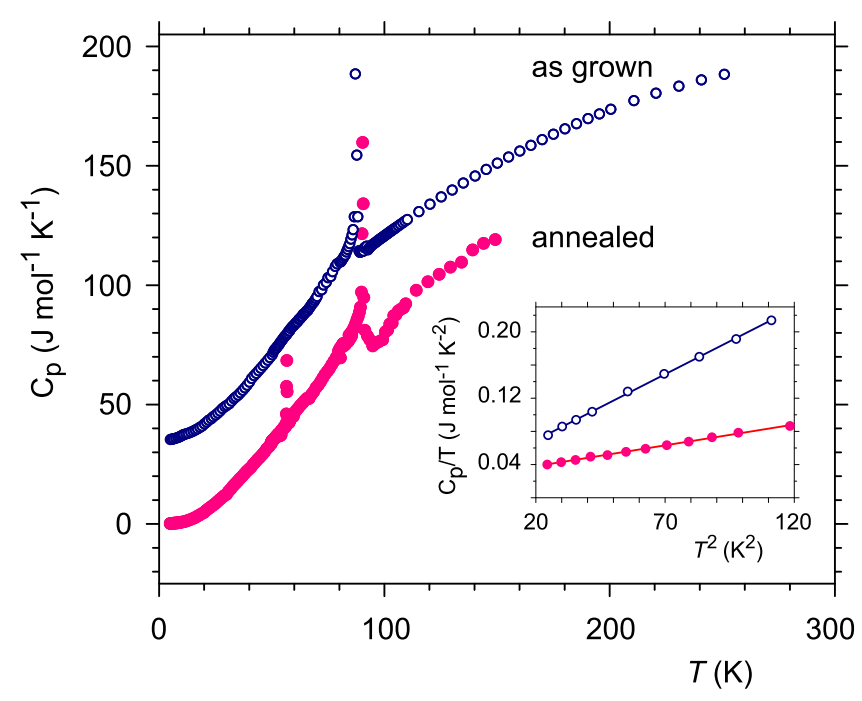

FIG. 2. Temperature dependence $(5-250 \mathrm{~K})$ of the specific heat $\left(C_{p}\right)$ of $\mathrm{CuV}_{2} \mathrm{~S}_{4}$, as measured on an as-grown crystal (blue open circles) and on the same crystal after annealing (red filled dots). Data for the as-grown crystal have been offset by $35 \mathrm{~J} \mathrm{~mol}^{-1} \mathrm{~K}^{-1}$. The inset shows $C_{p} / T$ at low temperatures. The linear fit resulted in $\gamma_{\text {asgr }}=37.6(9)$ and $\gamma_{\mathrm{ann}}=28.0(4) \mathrm{mJ} \mathrm{mol}^{-1} \mathrm{~K}^{-2}$ for the as-grown and annealed samples, respectively.

coefficient, $\gamma$, is found to be in good agreement with the value reported earlier (Fig. 2) [21,22,28]. This again implies that the crystal is of very good quality.

The magnetic susceptibility $(\chi)$ was measured with a commercial SQUID magnetometer (MPMS 7, Quantum Design, USA) in the temperature range 5-300 K. Data were collected while cooling the sample and again while heating the sample, both for the as-grown and annealed state. Anomalies in $\chi(T)$ are found for both transitions in all four curves, while transitions appear sharper for the annealed state (Fig. 3). The values of the susceptibility and the temperature dependence behavior are very similar to the previous reports $[21,22,28]$.

\section{Results}

\section{A. Structural phase transitions}

Based on anomalies observed in the temperature dependencies of transport properties, magnetic properties, and thermodynamic properties, Okada et al. [24] have identified two phase transitions for $\mathrm{CuV}_{2} \mathrm{~S}_{4}$, at temperatures of $T_{\mathrm{CDW}}=$ $91.5 \mathrm{~K}$ and $T_{c 2}=56 \mathrm{~K}$, respectively. These results are in agreement with the present data on the annealed state, for which we have observed anomalies in the temperature dependencies of the electrical resistivity (Fig. 1), specific heat (Fig. 2), and magnetic susceptibility (Fig. 3) at both transitions. The sharpest anomalies are found for the specific heat, resulting in transition temperatures $T_{\mathrm{CDW}}=90.37 \mathrm{~K}$ and $T_{c 2}=T_{\text {lock-in }}=56.68 \mathrm{~K}$. The up-turn of the resistivity at $T_{\mathrm{CDW}}$, the smaller value of the magnetic susceptibility in the CDW state, and the $\lambda$-shaped anomaly in the specific heat are all in agreement with a CDW transition at $T_{\mathrm{CDW}}$.

Employing an annealed single crystal, we could correlate these transitions with the development of an incommensurate CDW below $T_{\mathrm{CDW}}$ and a lock-in transition at $T_{c 2}=T_{\text {lock-in }}$ 


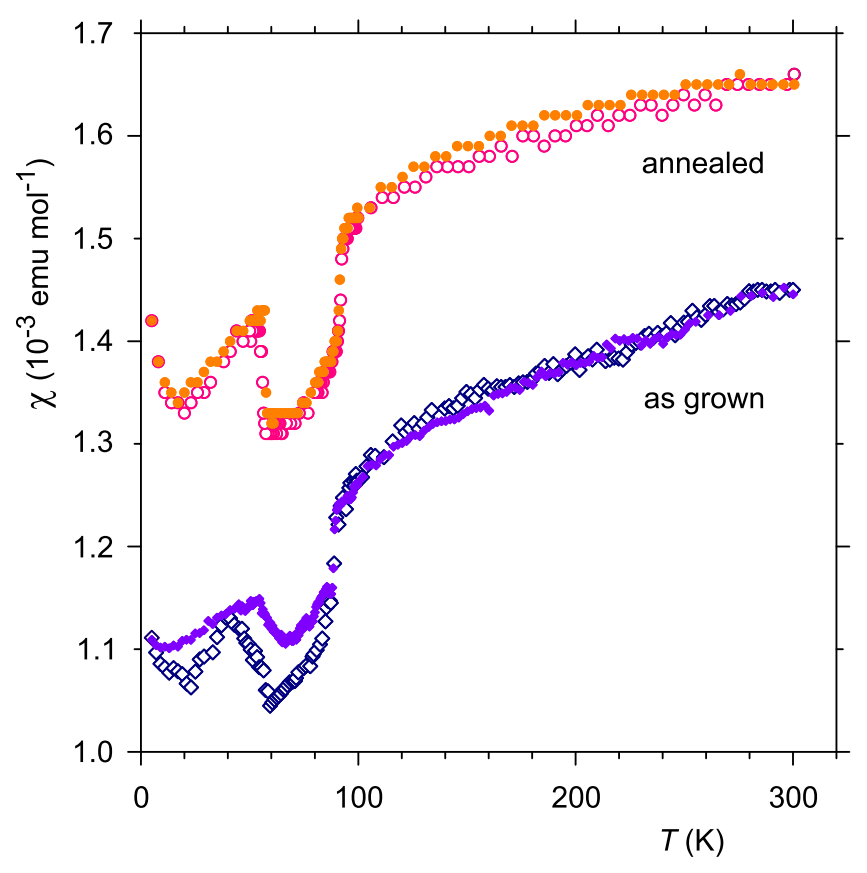

FIG. 3. Temperature dependence $(5-300 \mathrm{~K})$ of the magnetic susceptibility $(\chi)$ of $\mathrm{CuV}_{2} \mathrm{~S}_{4}$. Measured on an annealed crystal upon cooling (red open circles) and heating (orange filled dots), and measured on an as-grown crystal upon cooling (blue open diamonds) and heating (violet filled diamonds).

(Table I). The present diffraction data do not give accurate values for the transition temperatures, but they are in agreement with values obtained from transport properties. The components of the modulation wave vectors at 20 and $40 \mathrm{~K}$ seem to deviate from $2 / 3$. However, structure refinements (see below) show that a threefold supercell model gives a substantially better fit to the diffraction data than a model with an incommensurate modulation does, thus providing definite evidence for the commensurate character of the modulation in the lock-in phase below $T_{c 2}$. These features place the behavior of $\mathrm{CuV}_{2} \mathrm{~S}_{4}$ on par with many other aperiodic crystals, where an incommensurate phase transition is followed by a lockin transition at lower temperatures. Unique to $\mathrm{CuV}_{2} \mathrm{~S}_{4}$ is the sluggish character of the lock-in transition. Upon initial cooling from 80 to $20 \mathrm{~K}$, it took about four hours at $20 \mathrm{~K}$ for the transition toward the commensurate phase to complete.

TABLE I. Temperature dependence of the lattice parameters $a_{F}$, $c_{F}$, and $\gamma_{F}$, the volume $V$ of the unit cell, and the component $\sigma$ of the modulation wave vector $\mathbf{q}=(\sigma, \sigma, 0)$ for crystal A (annealed). See Sec. S1 of [32] for the $F$-centered setting of $F$-centered cubic and $I$-centered orthorhombic lattices.

\begin{tabular}{|c|c|c|c|c|c|}
\hline$T(\mathrm{~K})$ & $a_{F}(\AA)$ & $c_{F}(\AA)$ & $\gamma_{F}(\operatorname{deg})$ & $\sigma$ & $V\left(\AA^{3}\right)$ \\
\hline 20 & $9.7592(3)$ & $9.7576(4)$ & $89.993(2)$ & $0.6733(3)$ & $929.32(6)$ \\
\hline 40 & $9.7743(4)$ & $9.7726(5)$ & $90.012(3)$ & $0.6702(4)$ & $933.63(8)$ \\
\hline 60 & $9.7728(14)$ & $9.7738(13)$ & $89.996(4)$ & $0.7370(3)$ & $933.48(26)$ \\
\hline 80 & $9.7711(4)$ & $9.7664(4)$ & $89.987(2)$ & $0.7517(2)$ & $932.44(8)$ \\
\hline 90 & $9.7762(2)$ & $9.7736(4)$ & $89.984(2)$ & $0.7595(3)$ & $933.48(26)$ \\
\hline 250 & $9.8023(5)$ & & cubic lattice & & $941.87(15)$ \\
\hline
\end{tabular}

TABLE II. Temperature dependence of the lattice parameters $a_{F}$, $c_{F}$, and $\gamma_{F}$, the volume $V$ of the unit cell, and the component $\sigma$ of the modulation wave vector $\mathbf{q}=(\sigma, \sigma, 0)$ for crystal B (as-grown). See Sec. S1 of [32] for the $F$-centered setting of $F$-centered cubic and $I$-centered orthorhombic lattices.

\begin{tabular}{lccccc}
\hline \hline$T(\mathrm{~K})$ & $a_{F}(\AA)$ & $c_{F}(\AA)$ & $\gamma_{F}(\mathrm{deg})$ & $\sigma$ & $V\left(\AA^{3}\right)$ \\
\hline 80 & $9.7749(3)$ & $9.7588(5)$ & $89.945(3)$ & $0.7625(2)$ & $932.45(8)$ \\
81 & $9.7748(3)$ & $9.7596(4)$ & $89.961(3)$ & $0.7622(2)$ & $932.50(7)$ \\
82 & $9.7742(3)$ & $9.7596(5)$ & $89.958(3)$ & $0.7625(2)$ & $932.38(8)$ \\
83 & $9.7741(3)$ & $9.7591(5)$ & $89.960(2)$ & $0.7629(2)$ & $932.29(7)$ \\
84 & $9.7715(4)$ & $9.7587(5)$ & $89.959(3)$ & $0.7659(2)$ & $931.79(10)$ \\
85 & $9.7667(4)$ & $9.7654(5)$ & $89.994(3)$ & & $931.50(7)$ \\
87 & $9.7666(4)$ & $9.7669(5)$ & $90.002(4)$ & & $931.64(8)$ \\
88 & $9.7680(3)$ & $9.7659(5)$ & $89.994(3)$ & & $931.81(7)$ \\
89 & $9.7670(2)$ & $9.7653(4)$ & $89.981(3)$ & & $931.54(5)$ \\
91 & $9.7653(3)$ & $9.7635(5)$ & $89.987(4)$ & & $931.06(7)$ \\
92 & $9.7650(3)$ & $9.7631(4)$ & $90.003(4)$ & & $930.96(6)$ \\
93 & $9.7643(3)$ & $9.7636(5)$ & $89.988(3)$ & & $930.87(8)$ \\
94 & $9.7656(4)$ & $9.7630(4)$ & $89.996(3)$ & & $931.08(8)$ \\
95 & $9.7655(3)$ & $9.7643(5)$ & $90.009(4)$ & & $931.19(7)$ \\
\hline \hline
\end{tabular}

For the as-grown crystal $\mathrm{P}$, anomalies in the temperature dependencies of the electrical resistivity (Fig. 1) and magnetic susceptibility (Fig. 3) are again found at both transitions. However, they appear broadened and less pronounced, especially for the lock-in transition. The temperature dependence of the specific heat has a sharp anomaly at $T_{\mathrm{CDW}}^{\mathrm{asgr}}=87.08 \mathrm{~K}$ (Fig. 2), which value is $3.3 \mathrm{~K}$ lower than for the annealed state. The specific heat of the as-grown crystal $\mathrm{P}$ does not reveal any anomaly at the lock-in transition, suggesting that as-grown material does not proceed through the lock-in transition.

These results are corroborated by diffraction experiments. The SXRD experiment on crystal B (not annealed) revealed the CDW transition to take place at $84.5 \pm 0.5 \mathrm{~K}$ (Table II). The intensities of the satellite reflections as well as the magnitudes of the major modulation amplitudes of the refined structure models are in agreement with a second-order phase transition, although there are too few data points close to $T_{\mathrm{CDW}}$ for the determination of a critical exponent (Fig. 4). The transition of as-grown crystals is at a substantially lower temperature than the transition of annealed crystal A, where we have found by SXRD that the CDW phase exists at $90 \mathrm{~K}$. It appears that as-grown material goes into an undercooled state of the periodic phase, despite the fact that the CDW transition is of second order. The transition is inhibited by the "disorder" proposed by Horny et al. [28].

The lock-in transition at about $50 \mathrm{~K}$ has been observed upon initial cooling of as-grown crystal C. At $T=4.2 \mathrm{~K}$, satellite reflections appeared that could be described by the commensurate modulation wave vector with $\sigma=\frac{2}{3}$, while the original set of incommensurate satellite reflections remained (Table III). This observation showed that the lock-in transition has proceeded in only part of crystal C. Heating toward $35 \mathrm{~K}$ restored the incommensurate state, while subsequent cooling, as well as cooling of crystals D and E, never produced the commensurate state. Instead, we observe a decrease in the length of the incommensurate modulation wave vector toward lower temperatures (Fig. 5). Together, these results 


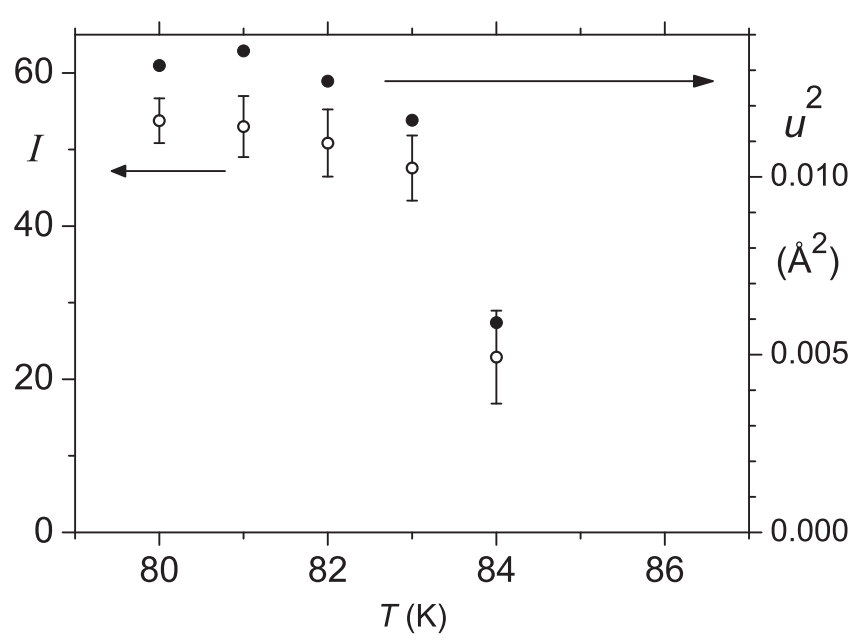

FIG. 4. Temperature dependence near the CDW transition of the intensity $(I)$ of the satellite Bragg reflection $(10,12,0,-1)$ (open circles; left scale), and square of the magnitude $(u)$ of the modulation amplitude of atom V1 (filled circles; right scale), as determined for crystal B. Notice the perfect scaling between these two quantities. The satellite intensity and modulation are zero for $85 \mathrm{~K}$ and higher temperatures.

TABLE III. Temperature dependence of the lattice parameters $a_{F}, c_{F}$, and $\gamma_{F}$, the volume $V$ of the unit cell, and the component $\sigma$ of the modulation wave vector $\mathbf{q}=(\sigma, \sigma, 0)$ for crystals $\mathrm{C}, \mathrm{D}$, and E (as-grown). See Sec. S1 of [32] for the $F$-centered setting of $F$-centered cubic and $I$-centered orthorhombic lattices.

\begin{tabular}{lccccl}
\hline \hline$T(\mathrm{~K})$ & $a_{F}(\AA)$ & $c_{F}(\AA)$ & $\gamma_{F}(\mathrm{deg})$ & $\sigma$ & $V\left(\AA^{3}\right)$ \\
\hline \multicolumn{2}{l}{ Crystal C } & & & & \\
$4.20^{\mathrm{a}}$ & $9.7746(3)$ & $9.7752(4)$ & $89.999(3)$ & $0.6593(8)$ & $933.96(7)$ \\
& & & & $0.7598(7)$ & \\
$4.4^{\mathrm{b}}$ & $9.7672(4)$ & $9.7660(6)$ & $89.989(4)$ & $0.7290(5)$ & $931.54(10)$ \\
$25^{\mathrm{c}}$ & $9.7754(4)$ & $9.7705(4)$ & $89.978(4)$ & $0.7496(6)$ & $933.12(6)$ \\
$35^{\mathrm{b}, \mathrm{d}}$ & $9.7759(4)$ & $9.7991(4)$ & $89.989(4)$ & $0.7532(6)$ & $934.57(9)$ \\
$35^{\mathrm{c}, \mathrm{d}}$ & $9.7739(4)$ & $9.7785(5)$ & $89.979(3)$ & $0.7561(5)$ & $933.69(9)$ \\
$39^{\mathrm{c}, \mathrm{e}}$ & $9.7745(6)$ & $9.7702(8)$ & $89.959(4)$ & $0.7585(4)$ & $933.45(14)$
\end{tabular}

Crystal D

$\begin{array}{llllll}5^{\mathrm{a}} & 9.7697(4) & 9.7627(4) & 89.987(2) & 0.7289(3) & 931.82(6) \\ 5^{\mathrm{b}, \mathrm{d}} & 9.7644(3) & 9.7612(4) & 89.988(3) & 0.7278(4) & 930.66(7) \\ 8^{\mathrm{c}, \mathrm{d}} & 9.7642(4) & 9.7614(3) & 89.985(4) & 0.7253(3) & 930.91(9) \\ 11^{\mathrm{c}, \mathrm{d}} & 9.7674(2) & 9.7656(4) & 89.998(4) & 0.7234(2) & 931.66(2) \\ 16^{\mathrm{b}} & 9.7718(4) & 9.7702(3) & 89.981(3) & 0.7476(3) & 932.93(8) \\ 20^{\mathrm{a}, \mathrm{e}} & 9.7710(3) & 9.7710(4) & 89.986(4) & 0.7586(3) & 933.13(9) \\ 30^{\mathrm{c}, \mathrm{d}} & 9.7779(4) & 9.7792(5) & 89.984(4) & 0.7489(3) & 934.96(9) \\ 35^{\mathrm{a}} & 9.7796(4) & 9.7641(5) & 89.949(2) & 0.7511(1) & 933.85(7)\end{array}$

Crystal E

$45^{\mathrm{d}} \quad 9.7701(3) \quad 9.7704(3) \quad 90.003(3) \quad 0.7508(2) \quad 932.60(6)$

\footnotetext{
${ }^{\mathrm{a}}$ First cooling.

${ }^{\mathrm{b}}$ Second cooling.

${ }^{\mathrm{c}}$ Third cooling.

${ }^{\mathrm{d}}$ Structure refinements not possible, because the formation of ice prevented the determination of intensities of Bragg reflections.

${ }^{\text {e}}$ Too few satellite reflections for refinement of the modulated structure.
}

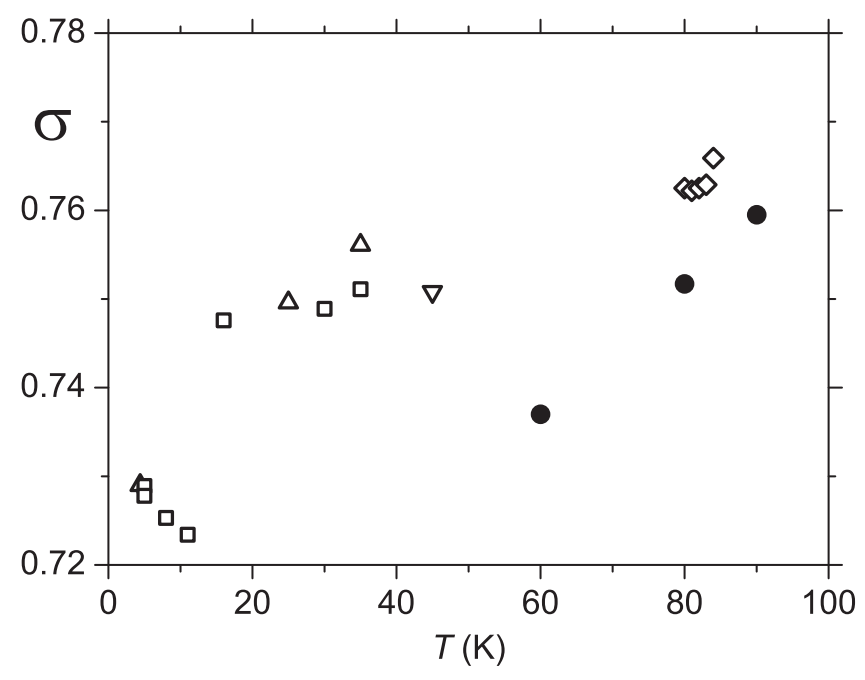

FIG. 5. Temperature dependence of the incommensurate component $\sigma$ of the modulation wave vector. Filled circles for crystal A (annealed). Open symbols for as-grown crystals, B (diamonds), C (up triangles), D (squares), and E (down triangle).

can be explained by modifications to the CDW state of asgrown crystals, which take place around the temperature of the lock-in transition, but that fail to develop long-range order. That is, the lock-in transition as such does not take place in as-grown crystals, despite anomalies observed in the temperature dependencies of the electrical resistivity, $\rho(T)$, and the magnetic susceptibility, $\chi(T)$ (also see Fig. 6).

For the annealed crystal, the incommensurate component $\sigma$ goes through the rational value $\frac{3}{4}$ upon cooling through about $80 \mathrm{~K}$ (Table I). For the as-grown crystals, the behavior is not exactly reproducible, but the component $\sigma$ again passes $\frac{3}{4}$, with a tendency toward a value of 0.725 at very low temperatures (Table III). These observations are in agreement with Kawaguchi et al. [26], who reported observing the same

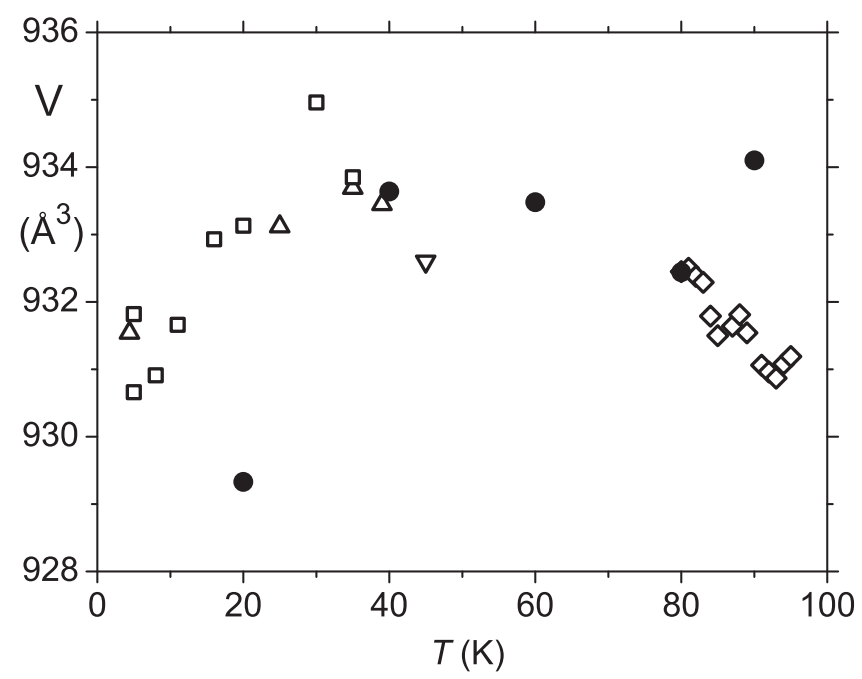

FIG. 6. Temperature dependence of the volume of the $F$ centered unit cell. Filled circles for crystal A (annealed). Open symbols for as-grown crystals, B (diamonds), C (up triangles), D (squares), and $\mathrm{E}$ (down triangle). 


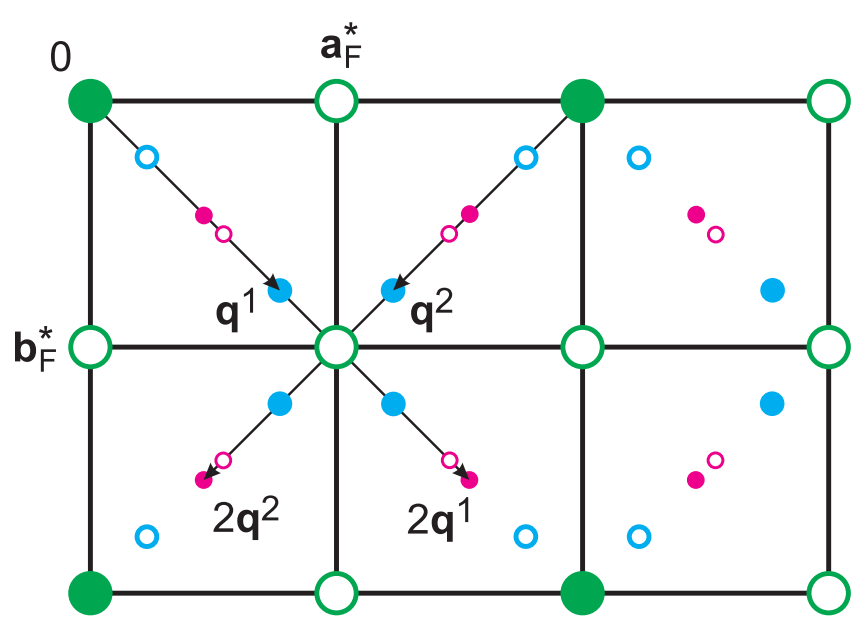

FIG. 7. Schematic representation of the $\left(\mathbf{a}_{F}^{*}, \mathbf{b}_{F}^{*}\right)$ plane of the diffraction pattern of incommensurate $\mathrm{CuV}_{2} \mathrm{~S}_{4}$ below $T_{\mathrm{CDW}}$. Open circles indicate the positions of reflections that are extinct due to the $F$ centering. Positions of second-order satellites are indicated, but these reflections have not been observed.

modulated crystal structures at 70 and $20 \mathrm{~K}$. Furthermore, they might explain the results of Fleming et al. [22], who found a presumed lock-in transition at $75 \mathrm{~K}$ toward the commensurate value $\frac{3}{4}$. Here it is noticed that the modulation wave vector $\mathbf{q}_{c}=\left(\frac{2}{3}, \frac{2}{3}, 0\right)$ describes a threefold superstructure, because $3 \mathbf{q}_{c}=(2,2,0)$ is a reciprocal-lattice vector of the basic lattice, while $\mathbf{q}=\left(\frac{3}{4}, \frac{3}{4}, 0\right)$ describes an eightfold superstructure, because $4 \mathbf{q}=(3,3,0)$ is a forbidden reciprocal point of the $F$-centered lattice, and only $8 \mathbf{q}$ is a reciprocallattice vector. Apparently, an eightfold supercell relation is of too high order for inducing a lock-in transition toward the value $\frac{3}{4}$.

\section{B. Diffraction symmetry and lattice distortion}

One of the open questions is the symmetry of the CDW phase, where the possibility must be considered that the development of the modulation is accompanied by a lowering of the point symmetry of crystal structure. The cubic space group $F d \overline{3} m$ (No. 227 [33]) has the maximum tetragonal subgroup I4 $/$ amd (No. 141), the latter of which has the orthorhombic subgroups Fddd (No. 70) and Imma (No. 74). Kawaguchi et al. [26] established noncentrosymmetric Imm 2 symmetry for the incommensurate CDW phase, with superspace group $\operatorname{Imm} 2(\sigma 00) 000$ [No. 44.1.12.4; standard setting $\operatorname{I2mm}(00 \sigma) 000$ [34]].

The present SXRD data allow for further tests of the lattice symmetry. In particular, the cubic lattice is approximately preserved in the CDW phase, because there is no obvious splitting of main reflections. Up to 12 satellite reflections around each main reflection can be described by six symmetry equivalent modulation wave vectors (Tables S3 and S4 in [32]). A tetragonal CDW structure would involve four of the 12 satellite reflections that are described by two modulation wave vectors (Fig. 7). The other satellite reflections then are due to threefold twinning. Three domains exist, with the tetragonal fourfold axis along one of $\mathbf{a}_{F}, \mathbf{b}_{F}$, and $\mathbf{c}_{F}$ of the $F$-centered unit cell, respectively. The point symmetry
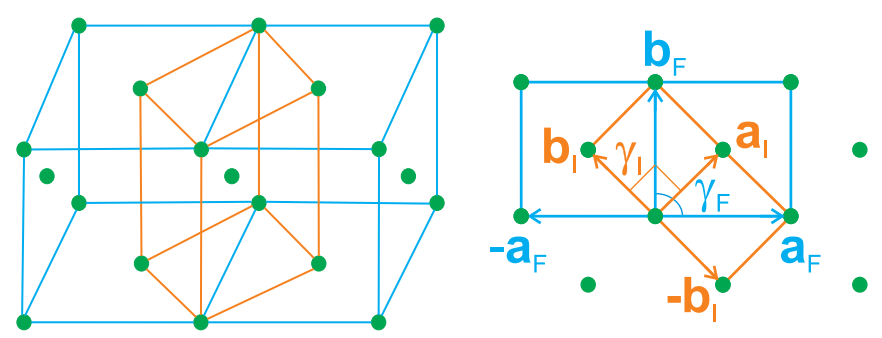

FIG. 8. Relation between the $F$-centered (blue) and $I$-centered (orange) unit cells for the pseudocubic $F$-centered lattice.

of the main Bragg reflections of such a twinned crystal is orthorhombic $\mathrm{mmm}$, whereby the mirror planes refer to mirror planes perpendicular to the coordinate axes of the $F$-centered unit cell. However, satellites from different domains are completely separated, so that the full $4 / \mathrm{mmm}$ symmetry would apply to them. Averaging the diffraction data according to these symmetries resulted in $R_{\mathrm{int}}=0.487$ for the satellite reflections, which provides definite proof that the CDW phase does not have tetragonal symmetry nor orthorhombic $F d d d$ symmetry (Table S5 in [32]).

Orthorhombic Imm 2 symmetry refers to mirror planes perpendicular to the face diagonals of the $F$-centered unit cell and a twofold axis along the coordinate axis $\mathbf{c}_{F}$. Sixfold twinning combined with inversion twinning results in diffraction symmetry $\overline{1}$ of the overlapping main Bragg reflections. Each of the six pairs of satellite reflections at $\pm \mathbf{q}^{j}(j=1, \ldots, 6)$ has contributions from a pair of inversion domains (Tables S3 and S4 in [32]). Since Friedel's law is valid in a good approximation, satellite reflections should have $\mathrm{mmm}$ symmetry referring to the $I$-centered lattice. Averaging according to this symmetry resulted in $R_{\text {int }}=0.104$ for the satellite reflections, while $R_{\sigma}=0.117\left(R_{\sigma}\right.$ is the average of the standard uncertainty divided by the intensity). These values show that orthorhombic symmetry according to Immm is fulfilled within the accuracy of the data (Table S5 in [32]).

The $F$-centered unit cell (lattice parameters $a_{F}=b_{F} \neq$ $c_{F}$ and $\alpha_{F}=\beta_{F}=\gamma_{F}=90^{\circ}$ ) is a valid alternate setting for the $I$-centered tetragonal lattice with $a_{I}=b_{I}=a_{F} / \sqrt{2}$ and $c_{I}=c_{F}$ (Fig. 8) [35]. It is noticed here that an $F$-centered unit cell can also be chosen for the $I$-centered orthorhombic lattice. The orthorhombic distortion, $a_{I} \neq b_{I}$, of the $I$-centered unit cell is reflected in the $F$-centered unit cell by $\gamma_{F} \neq 90^{\circ}$, while $a_{F}=b_{F}=\sqrt{a_{I}^{2}+b_{I}^{2}}, c_{F}=c_{I}$, and $\alpha_{F}=\beta_{F}$ remain $90^{\circ}$ (see Sec. S1 of [32]). The ratio $\frac{c_{F}}{a_{F}}$ is thus a good measure for the tetragonal lattice distortion, while $\gamma_{F}$ is a good measure for the orthorhombic distortion (Fig. 9). The ratio $\frac{c_{F}}{a_{F}}$ varies irregularly over the different samples B-E. For crystal B, $\frac{c_{F}}{a_{F}} \approx 1$ for the cubic phase, while it is $\sim 0.998$ in the CDW phase. The latter values are comparable to those of Kawaguchi et al. [26] and Okada et al. [24] at $70 \mathrm{~K}$. These values suggest that previous work has studied as-grown crystalline material. Okada et al. [24] have reported an increasing distortion on decreasing temperature. We find different behavior for the as-grown crystals $\mathrm{C}-\mathrm{E}$, which have lattice distortions smaller than that of crystal B [Fig. 9(a)]. These disparate behaviors are again in agreement with samples in nonequilibrium states, 

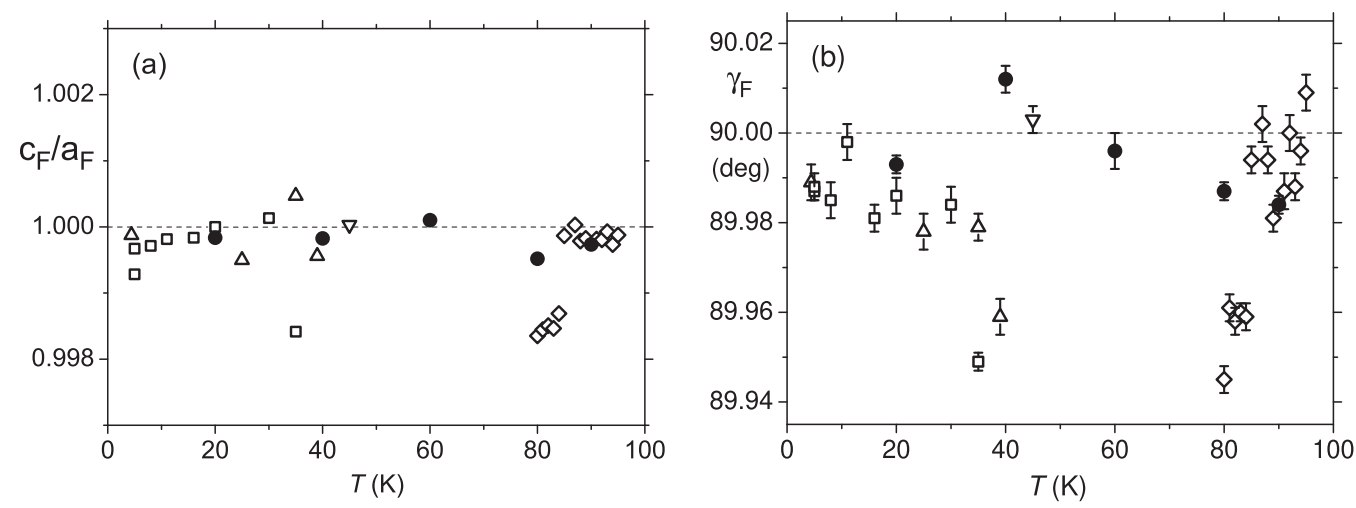

FIG. 9. Temperature dependence of (a) ratio $c_{F} / a_{F}$ of the lattice parameters $a_{F}$ and $c_{F}$, and (b) the lattice parameter $\gamma_{F}$. Both refer to the $F$-centered setting of the $I$-centered tetragonal or orthorhombic lattices. Filled circles for crystal A (annealed). Open symbols for as-grown crystals, B (diamonds), C (up triangles), D (squares), and E (down triangle).

where lattice defects may depend on the precise thermal history of each sample.

Further support for this interpretation comes from crystal A (annealed), for which we have obtained values within the range $0.9995<\frac{c_{F}}{a_{F}}<1.0001$. Apparently, the tetragonal lattice distortion (which is part of the orthorhombic distortion) is much smaller for equilibrium crystals than for the disordered crystals. The magnitude of this small distortion cannot be reliably extracted from our experiments.

A similar picture is obtained for the second part of the orthorhombic distortion, which is described by $\gamma_{F}$. The largest distortion is obtained for crystal B [Fig. 9(b)], with the value of $\gamma_{F} \approx 89.95^{\circ}$ equal to the values obtained by Kawaguchi et al. [26]. Okada et al. [24] report $a_{F}=b_{F}$ and $c_{F}$ and conclude toward a tetragonal lattice for 8-90 K. However, they do not report the value of $\gamma_{F}$, and might have mistakenly considered any deviations from $90^{\circ}$ as inaccuracies of their analysis instead of being the orthorhombic distortion toward the $I$-centered lattice. The present data on as-grown crystals $\mathrm{C}-\mathrm{E}$ suggest a decrease of the orthorhombic distortion upon cooling, whereas the annealed crystal A has nearly zero orthorhombic distortion, which apparently is the signature of equilibrated material.

\section{Crystal structures of the CDW and lock-in states}

The cubic spinel structure has been confirmed for annealed crystal A at $250 \mathrm{~K}$ and for as-grown crystal B at $95 \mathrm{~K}$. Structure refinements with the software JANA2006 [36] gave an excellent fit to the diffraction data [32].

A good fit to the diffraction data of the incommensurate CDW phase could only be achieved for superspace symmetry $\operatorname{Imm} 2\left(\begin{array}{ll}\sigma & 0\end{array}\right)$, in agreement with the observed diffraction symmetry (Sec. III B). Employing single-harmonic modulation functions (Eq. S8 in [32]), a good fit has been obtained to both main reflections and satellite reflections of crystal A at temperatures of 60, 80, and $90 \mathrm{~K}$ (Tables S2 and S6 of [32]), of crystal B at 80-85 K, of crystal C at 4.4 and $25 \mathrm{~K}$, and of crystal D at 5, 16, and $35 \mathrm{~K}$ (Tables S12- S15 of [32]). Structure models are similar between different crystals A-D, while a tendency is observed toward larger modulation amplitudes at lower temperatures. This result implies that annealed and as-grown material reach the same CDW state at low temperatures. Accordingly, the present analysis will be concentrated on the results for crystal A (annealed) at $80 \mathrm{~K}$.

The present modulation functions bear a great similarity to the first-order harmonics reported by Kawaguchi et al. [26], especially when considering the large standard uncertainties of $\sim 0.015 \AA$ for the latter Rietveld refinements against XRPD data [26]. The present refinements against SXRD data have resulted in much lower standard uncertainties (Table S10 in [32]). Kawaguchi et al. [26] also report modulation amplitudes of second harmonic, which are of larger magnitude than first-order harmonics. The largest amplitude is $B_{2, x}=B_{2, y}=$ 0.13(1) $\AA$ for atom V1. Amplitudes of this large magnitude should have led to strong second-order satellite reflections, which we do not observe. The second harmonic reported in [26] thus should be considered as an artifact of the Rietveld refinement. Second-order harmonics have been successfully introduced into the present refinements against SXRD data, despite the missing second-order satellites. However, resulting amplitudes are insignificant in view of their large standard uncertainties. Therefore, we propose modulation functions consisting of solely first-order harmonics as the final model.

Another major discrepancy between Kawaguchi et al.'s [26] model and the present model is that we find a significant orthorhombic distortion of the basic structure, especially for atom V2, while it is zero for atom V1. This structural feature has important consequences for understanding the CDW phase (see Sec. III D below).

The structure of the lock-in phase has been determined for crystal $\mathrm{A}$ at 20 and $40 \mathrm{~K}$. The lock-in phase is described by the same superspace symmetry $\operatorname{Imm} 2(\sigma 00)$ as applies to the incommensurate CDW. Structure refinements of the modulated structure against SXRD data resulted in a fit that is much better for the commensurate model than for an incommensurate model (Tables S6 and S7 in [32]).

\section{Vanadium clusters in the CDW state}

Band-structure calculations have shown that the Fermi level in $\mathrm{CuV}_{2} \mathrm{~S}_{4}$ is mainly composed of $3 d$ states of vanadium 


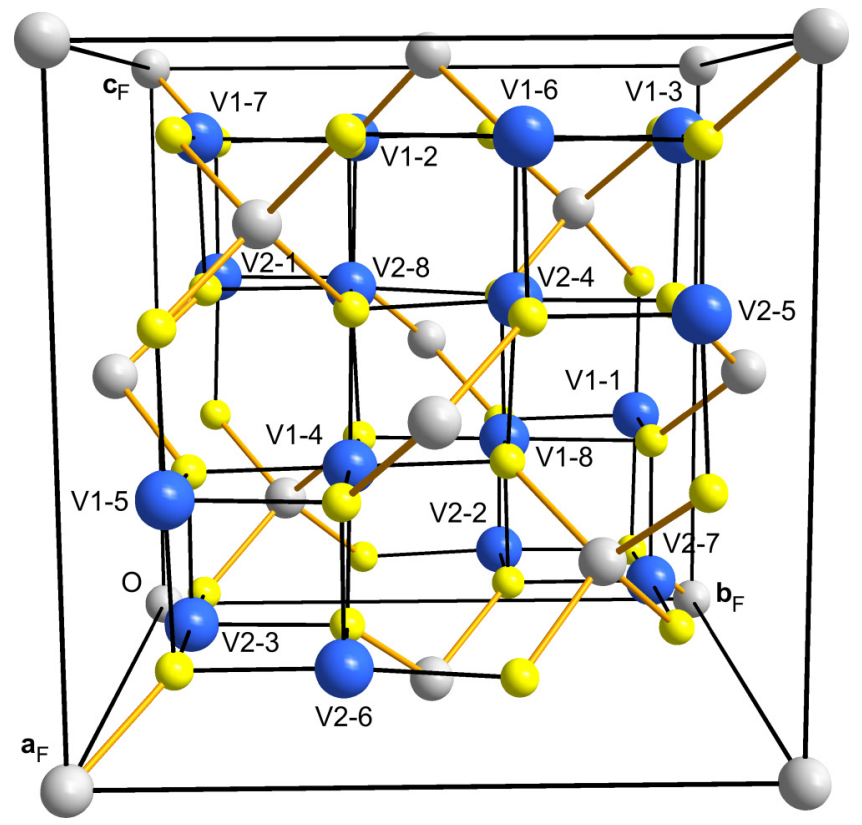

FIG. 10. Perspective view of the crystal structure of $\mathrm{CuV}_{2} \mathrm{~S}_{4}$. The basis vectors of the $F$-centered unit cell are indicated as $\mathbf{a}_{F}, \mathbf{b}_{F}$, and $\mathbf{c}_{F}=\mathbf{c}_{I}$. Labels V1-1, V2-1, and other labels indicate atoms equivalent by symmetry to $\mathrm{V} 1$ and $\mathrm{V} 2$, respectively. Blue is vanadium, gray is copper, and yellow is sulfur.

[37,38]. Therefore, it is a reasonable assumption that the origin of the CDW will be related to the modulation of the vanadium atoms. This idea is reinforced by the fact that the largest modulation amplitudes are the displacements of vanadium (Table S10). Furthermore, all short distances between metal atoms are between vanadium atoms, while copper atoms have isolated positions farther away from any other metal atom.

The orthorhombic crystal structure of $\mathrm{CuV}_{2} \mathrm{~S}_{4}$ contains chains of V2 atoms along $\mathbf{a}_{F}+\mathbf{b}_{F}$ (the orthorhombic axis $\mathbf{a}_{I}$ ) and chains of $\mathrm{V} 1$ atoms along $-\mathbf{a}_{F}+\mathbf{b}_{F}$ (the orthorhombic axis $\mathbf{b}_{I}$; see Fig. 10 and Sec. S1 in [32]). The modulation wave vector is parallel to $\mathbf{a}_{I}$, i.e., along the V2 chains.

The model by Kawaguchi et al. [26] involves dimerization of the V1 chains, while the incommensurate modulation of the interatomic distances along the V2 chains does not display clear features. It was thus proposed that the CDW is a commensurate CDW on the V1 chains, while interchain interactions would be responsible for the incommensurability of the modulation, in analogy to the situation for $\mathrm{SmNiC}_{2}$ [39].

The present structure model again involves dimerization of the V1 chains, albeit with double the period in [26], since the present model does not involve second-order harmonics (Fig. 11). We have presently found a major distortion of the average positions of the $\mathrm{V} 2$ atoms, a feature not present in the model in [26], and resulting in a dimerization of the basic structure of the V2 chains by $0.18 \AA$. Furthermore, the phase shift of the modulation between consecutive atoms along the V2 chain of $\sigma / 2 \approx 0.375$ results in a V2 chain containing dimers and trimers, and there is no compelling evidence favoring a CDW on the V1 chains.

An important difference between the cubic spinel structure and orthorhombic $\mathrm{SmNiC}_{2}$ is that the latter contains chains of
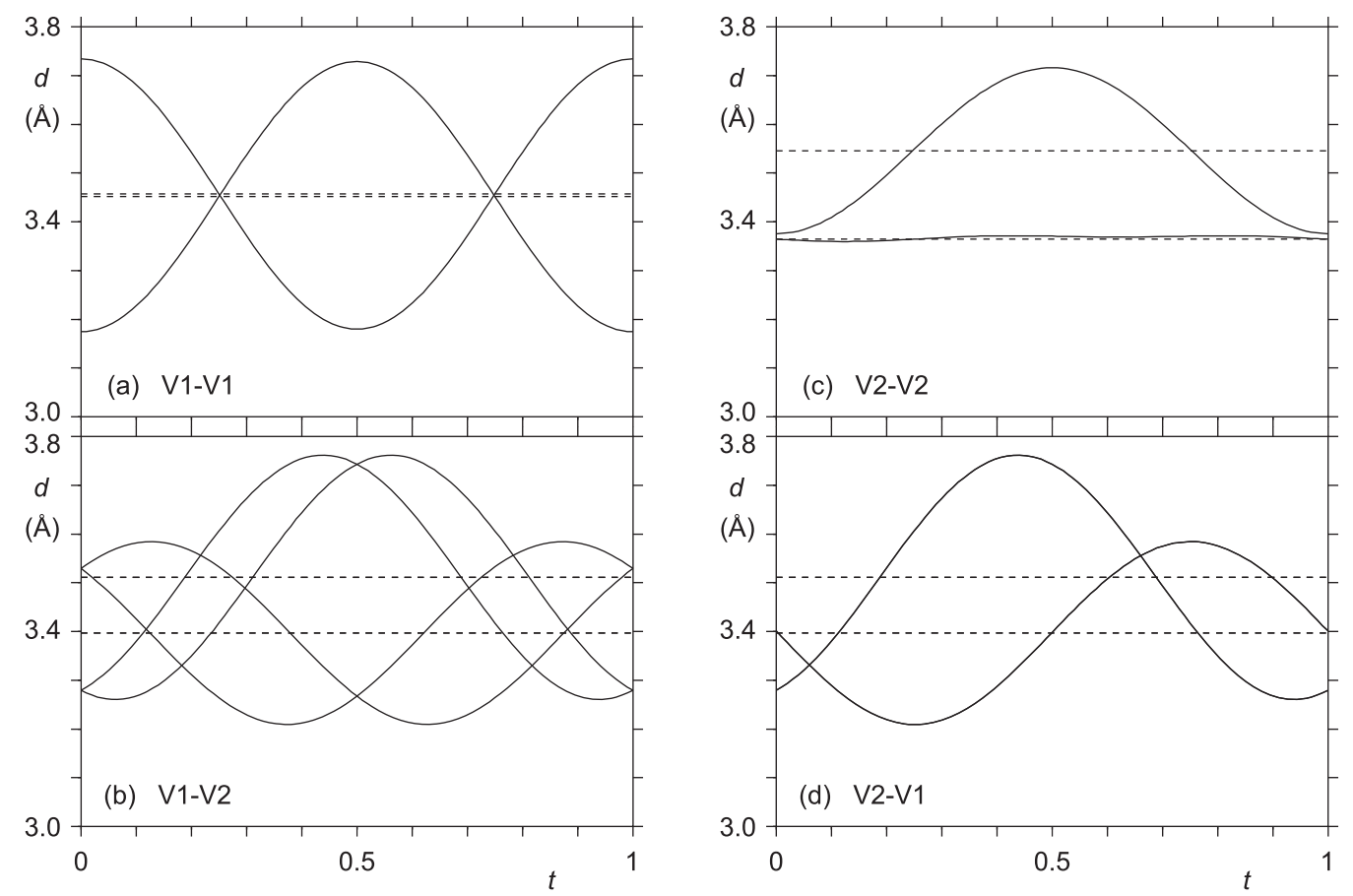

FIG. 11. $t$-plot of interatomic distances between one $\mathrm{V}$ atom and its six surrounding $\mathrm{V}$ atoms for the incommensurate CDW state of crystal $\mathrm{A}$ at $80 \mathrm{~K}$. Horizontal dashed lines indicate distances for the basic structure. (a),(b) The central atom is $\mathrm{V} 1$ at $(1 / 8,-1 / 8,3 / 8)$, which is part of $\mathrm{V}$-atom chains along $-\mathbf{a}_{F}+\mathbf{b}_{F}, \mathbf{b}_{F}+\mathbf{c}_{F}$, and $-\mathbf{a}_{F}+\mathbf{c}_{F}$. (c),(d) The central atom is $\mathrm{V} 2$ at $(1 / 8,1 / 8,5 / 8)$, which is part of $\mathrm{V}$-atom chains along $\mathbf{a}_{F}+\mathbf{b}_{F}, \mathbf{b}_{F}+\mathbf{c}_{F}$, and $\mathbf{a}_{F}+\mathbf{c}_{F}$. 

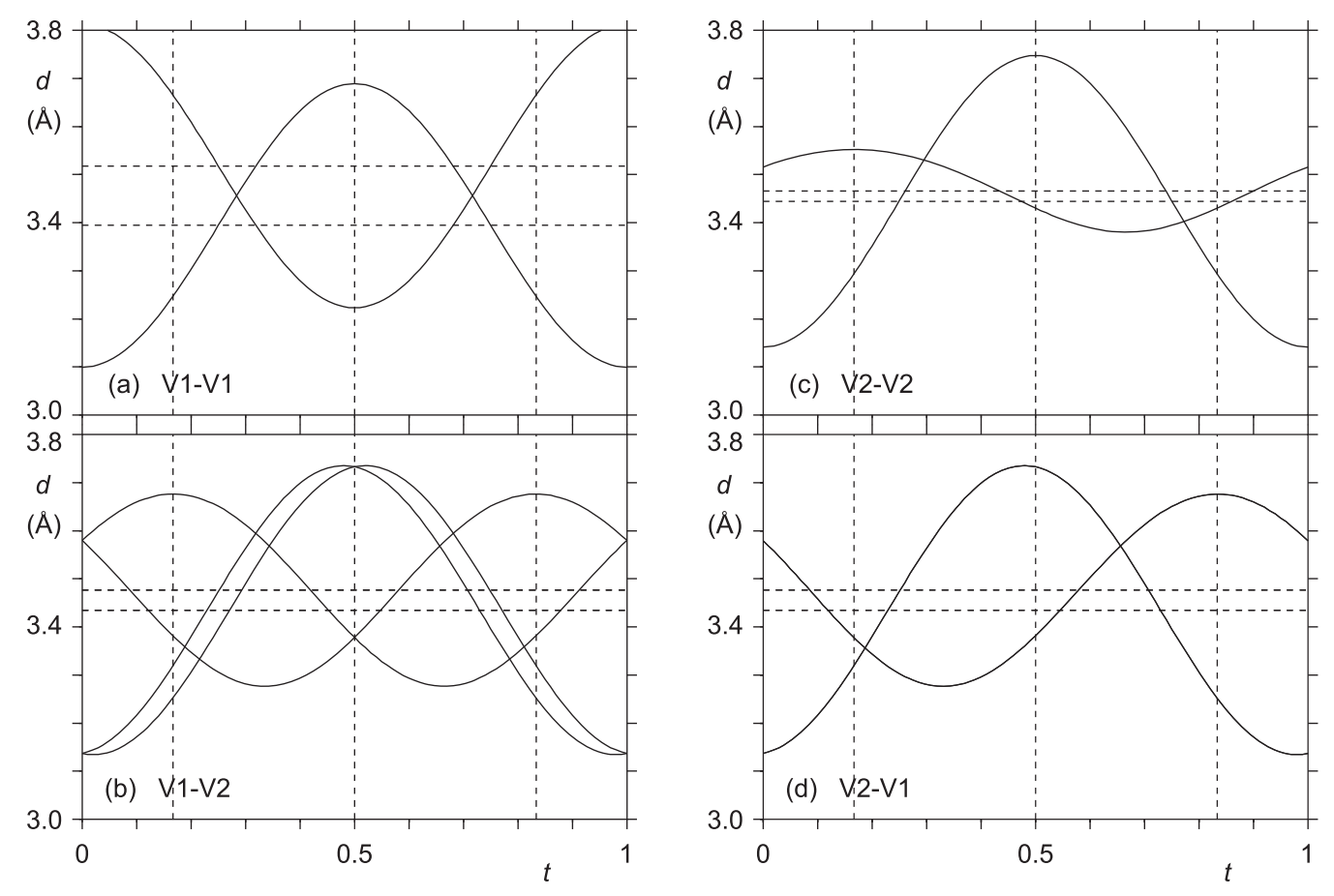

FIG. 12. $t$-plot of interatomic distances between one $\mathrm{V}$ atom and its six surrounding $\mathrm{V}$ atoms for the commensurately modulated structure of the lock-in state of crystal A at $40 \mathrm{~K}$. The threefold superstructure involves distances and atomic positions at $t$-values of $\frac{1}{6}, \frac{1}{2}$, and $\frac{5}{6}$, indicated by vertical dashed lines. Horizontal dashed lines indicate distances for the basic structure. (a),(b) The central atom is $\mathrm{V} 1$ at $(1 / 8,-1 / 8,3 / 8)$. (c),(d) The central atom is V2 at $(1 / 8,1 / 8,5 / 8)$. Distances are shown between the same atoms as have been used for Fig. 11.

$\mathrm{Ni}$ atoms along a single orthorhombic axis, while the spinel structure contains chains of $\mathrm{V}$ atoms along the six equivalent directions $\pm \mathbf{a}_{F}+\mathbf{b}_{F}, \pm \mathbf{a}_{F}+\mathbf{c}_{F}$, and $\pm \mathbf{b}_{F}+\mathbf{c}_{F}$. Referring to the $F$-centered setting of the orthorhombic Imm2 structure, each $\mathrm{V} 1$ atom is at the intersection of the $\mathrm{V} 1$ chain along $-\mathbf{a}_{F}+\mathbf{b}_{F}$, and two chains composed of alternating V1 and $\mathrm{V} 2$ atoms. For one V1 atom, these mixed chains are along $\mathbf{b}_{F}+\mathbf{c}_{F}$ and $-\mathbf{a}_{F}+\mathbf{c}_{F}$. The orthorhombic symmetry then implies that a second, symmetry-equivalent $\mathrm{V} 1$ atom is at the intersection of the V1 chain and two V1-V2 mixed chains, along $-\mathbf{b}_{F}+\mathbf{c}_{F}$ and $\mathbf{a}_{F}+\mathbf{c}_{F}$. Similarly, each V2 atom is at the intersection of the V2 chain along $\mathbf{a}_{F}+\mathbf{b}_{F}$, and two V2-V1 mixed chains, along $\mathbf{b}_{F}+\mathbf{c}_{F}$ and $\mathbf{a}_{F}+\mathbf{c}_{F}$. A second, symmetry-equivalent $\mathrm{V} 2$ atom is at the intersection of the V2 chain and two V2-V1 mixed chains, along $-\mathbf{b}_{F}+\mathbf{c}_{F}$ and $-\mathbf{a}_{F}+\mathbf{c}_{F} . t$-plots of $\mathrm{V}$-V interatomic distances then indicate that large modulations and short distances also involve V1V2 contacts (Fig. 11). Depending on $t$, extended clusters of vanadium atoms are formed, which are eventually interrupted by long distances due to the incommensurability of the modulation. Accordingly, we propose that the formation of 3D clusters is at the basis of the CDW in $\mathrm{CuV}_{2} \mathrm{~S}_{4}$.

Modulation amplitudes for the lock-in state are similar to but distinctly different from those for the incommensurate CDW phase. The distortion of the basic structure is much smaller for the lock-in state than for the incommensurate CDW. On the other hand, the commensurability determines that values of the modulation functions are physically relevant for only three values of their arguments. In the present structure models, those are the values of $t$ equal to $\frac{1}{6}, \frac{1}{2}$, and $\frac{5}{6}$ (vertical dashed lines in Fig. 12). Similar to the incommensurate structure, the lock-in state is characterized by extended clusters defined by short interatomic V-V distances.

\section{CONCLUSIONS}

$\mathrm{CuV}_{2} \mathrm{~S}_{4}$ possesses the cubic spinel structure at room temperature. We have established that the formation of an incommensurate $\mathrm{CDW}$ state at $T_{\mathrm{CDW}} \approx 91 \mathrm{~K}$ is followed by a lock-in transition toward a threefold superstructure at $T_{\text {lock-in }} \approx 50 \mathrm{~K}$. The phase transitions critically depend on the thermal history of the sample. As-grown samples are supposed to suffer from disorder and other lattice defects $[21,28]$. These defects do not show up in the diffraction of the spinel structure, but they are responsible for a suppression by a few $\mathrm{K}$ of $T_{\mathrm{CDW}}$ (Sec. III A). Despite the presence of anomalies around $T_{\text {lock-in }}$ in the temperature dependencies of the electrical resistivity and magnetic susceptibility of an asgrown crystal, corresponding superlattice reflections have not been observed in SXRD. It is worthwhile to recall that the temperature dependence of the heat capacity does not exhibit an anomaly for the lock-in transition in the as-grown crystal as well (Fig. 2). This implies that long-range order of the lock-in phase does not develop in as-grown material, but requires annealed crystals. Instead, the length of the incommensurate CDW wave vector continues to decrease toward the lowest temperatures in as-grown crystals (Fig. 5).

Both as-grown and annealed materials transform into the same, incommensurate CDW state, as is evidenced by the structure models at low temperatures for both types of crystal. The orthorhombic superspace symmetry of the CDW state is the same as proposed by Kawaguchi et al. [26]. However, 
structural parameters of the present models are much more accurate than those of the Rietveld refinement [26], and the present structure models are significantly different from that in [26].

The largest modulation amplitudes and shortest interatomic distances between metal atoms are found for vanadium atoms. They suggest that the formation of the CDW is governed by the vanadium atoms, in agreement with band-structure calculations for the spinel phase, which indicate $3 d$ vanadium states at the Fermi level $[37,38]$. The incommensurate CDW state can be characterized by the formation of extended vanadium clusters, which are interrupted by long V-V distances due to the incommensurability. In the lock-in phase, a connected path of short V-V distances percolates throughout the crystal in all directions. The lowering toward orthorhombic symmetry appears to be responsible for the precise pattern of modulations and short and long V-V distances. The actual orthorhombic lattice distortion-visible for some as-grown material-is nearly zero within standard uncertainties for the annealed crystal (Fig. 9).

\section{ACKNOWLEDGMENTS}

Single crystals of $\mathrm{CuV}_{2} \mathrm{~S}_{4}$ have been synthesized by Kerstin Küspert at the Laboratory of Crystallography in Bayreuth. We thank Mariia Anurova for assistance with initial diffraction experiments. We thank Dmitry Chernyshov (SNBL) for assistance with the synchrotron experiments at beam line BM01 of the SNBL at the ESRF in Grenoble, France. This research has been funded by the Deutsche Forschungsgemeinschaft (DFG, German Research Foundation)-265092781, and the Alexander-von-Humboldt Foundation within their research-group linkage program.
[1] G. Gruner, Density Waves in Solids (Addison-Wesley, Reading, MA, 1994).

[2] P. Monceau, Electronic crystals: an experimental overview, Adv. Phys. 61, 325 (2012).

[3] Physics and Chemistry of Low-dimensional Inorganic Conductors, edited by C. Schlenker, J. Dumas, M. Greenblatt, and S. van Smaalen, Vol. 354 of NATO ASI Series B: Physics (Plenum, New York, 1996).

[4] J. Chaussy, P. Haen, J. C. Lasjaunias, P. Monceau, G. Waysand, A. Waintal, A. Meerschaut, P. Molinie, and J. Rouxel, Phase transitions in $\mathrm{NbSe}_{3}$, Solid State Commun. 20, 759 (1976).

[5] M. D. Johannes and I. I. Mazin, Fermi surface nesting and the origin of charge density waves in metals, Phys. Rev. B 77, 165135 (2008).

[6] X. Zhu, Y. Cao, J. Zhang, E. W. Plummer, and J. Guo, Classification of charge density waves based on their nature, Proc. Natl. Acad. Sci. (USA) 112, 2367 (2015).

[7] T. Ritschel, J. Trinckauf, K. Koepernik, B. Buchner, M. V. Zimmermann, H. Berger, Y. I. Joe, P. Abbamonte, and J. Geck, Orbital textures and charge density waves: towards orbitronics in transition metal dichalcogenides, Nat. Phys. 11, 328 (2015).

[8] C.-W. Chen, J. Choe, and E. Morosan, Charge density waves in strongly correlated electron systems, Rep. Prog. Phys. 79, 084505 (2016).

[9] S. Ramakrishnan and S. van Smaalen, Unusual ground states in $R_{5} T_{4} X_{10}(R=$ rare earth; $T=\mathrm{Rh}, \mathrm{Ir} ;$ and $X=\mathrm{Si}, \mathrm{Ge}, \mathrm{Sn})$ : a review, Rep. Prog. Phys. 80, 116501 (2017).

[10] S. Shimomura, C. Hayashi, G. Asaka, N. Wakabayashi, M. Mizumaki, and H. Onodera, Charge-Density-Wave Destruction and Ferromagnetic Order in $\mathrm{SmNiC}_{2}$, Phys. Rev. Lett. 102, 076404 (2009).

[11] S. Shimomura, C. Hayashi, N. Hanasaki, K. Ohnuma, Y. Kobayashi, H. Nakao, M. Mizumaki, and H. Onodera, Multiple charge density wave transitions in the antiferromagnets $R \mathrm{NiC}_{2}$ ( $R=$ Gd, Tb), Phys. Rev. B 93, 165108 (2016).

[12] K. K. Kolincio, M. Roman, M. J. Winiarski, J. StrychalskaNowak, and T. Klimczuk, Magnetism and charge density waves in $R \mathrm{NiC}_{2}(R=\mathrm{Ce}, \mathrm{Pr}, \mathrm{Nd})$, Phys. Rev. B 95, 235156 (2017).

[13] M. Roman, J. Strychalska-Nowak, T. Klimczuk, and K. K. Kolincio, Extended phase diagram of $R \mathrm{NiC}_{2}$ family: Linear scaling of the Peierls temperature, Phys. Rev. B 97, 041103(R) (2018).

[14] Y. Otomo, K. Iwasa, K. Suyama, K. Tomiyasu, H. Sagayama, R. Sagayama, H. Nakao, R. Kumai, and Y. Murakami, Chiral crystal-structure transformation of $R_{3} \mathrm{Co}_{4} \mathrm{Sn}_{13}(R=\mathrm{La}$ and Ce), Phys. Rev. B 94, 075109 (2016).

[15] C. N. Kuo, C. W. Tseng, C. M. Wang, C. Y. Wang, Y. R. Chen, L. M. Wang, C. F. Lin, K. K. Wu, Y. K. Kuo, and C. S. Lue, Lattice distortion associated with Fermi-surface reconstruction in $\mathrm{Sr}_{3} \mathrm{Rh}_{4} \mathrm{Sn}_{13}$, Phys. Rev. B 91, 165141 (2015).

[16] H. T. Wang, M. K. Srivastava, C. C. Wu, S. H. Hsieh, Y. F. Wang, Y. C. Shao, Y. H. Liang, C. H. Du, J. W. Chiou, C. M. Cheng, J. L. Chen, C. W. Pao, J. F. Lee, C. N. Kuo, C. S. Lue, M. K. Wu, and W. F. Pong, Electronic and atomic structures of the $\mathrm{Sr}_{3} \mathrm{Ir}_{4} \mathrm{Sn}_{13}$ single crystal: A possible charge density wave material, Sci. Rep. 7, 40886 (2017).

[17] Y. K. Kuo, K. M. Sivakumar, J. Y. Lin, C. N. Kuo, and C. S. Lue, First-order phase transition in the ternary sulfide $\mathrm{NiV}_{2} \mathrm{~S}_{4}$, J. Phys.: Condens. Matter 19, 216210 (2007).

[18] R. N. Shelton, L. S. Hausermann-Berg, P. Klavins, H. D. Yang, M. S. Anderson, and C. A. Swenson, Electronic phase transition and partially gapped Fermi surface in superconducting $\mathrm{Lu}_{5} \mathrm{Ir}_{4} \mathrm{Si}_{10}$, Phys. Rev. B 34, 4590 (1986).

[19] E. Morosan, H. W. Zandbergen, B. S. Dennis, J. W. G. Bos, Y. Onose, T. Klimczuk, A. P. Ramirez, N. P. Ong, and R. J. Cava, Superconductivity in $\mathrm{Cu}_{x} \mathrm{TiSe}_{2}$, Nat. Phys. 2, 544 (2006).

[20] F. J. DiSalvo and J. V. Waszczak, Magnetic properties of copper chalcogenide spinels, Phys. Rev. B 26, 2501 (1982).

[21] R. Horny, Struktur und transporteigenschaften des elektronisch korrelierten thiospinells $\mathrm{CuV}_{2} \mathrm{~S}_{4}$, Ph.D. thesis, University of Augsburg, Augsburg, Germany (2005).

[22] R. M. Fleming, F. J. DiSalvo, R. J. Cava, and J. V. Waszczak, Observation of charge-density waves in the cubic spinel structure $\mathrm{CuV}_{2} \mathrm{~S}_{4}$, Phys. Rev. B 24, 2850 (1981).

[23] Fleming et al. [22] incorrectly report "reduced" modulation wave vectors $\mathbf{q}_{F}=\left(\frac{1}{4}-\delta\right)(1,1,0)$ and $\mathbf{q}_{F}^{\prime}=\left(\frac{1}{3}-\right.$ $\left.\delta^{\prime}\right)(1,1,0)$, which are not equivalent to $\mathbf{q}=(1,1,0)-\mathbf{q}_{F}$ and $\mathbf{q}^{\prime}=(1,1,0)-\mathbf{q}_{F}^{\prime}$, because $(1,1,0)$ is a forbidden reflection of the F-centered lattice. 
[24] H. Okada, K. Koyama, and K. Watanabe, Two-step structural modulations and Fermi liquid state in spinel compound $\mathrm{CuV}_{2} \mathrm{~S}_{4}$, J. Phys. Soc. Jpn 73, 3227 (2004).

[25] H. Okada, K. Koyama, and K. Watanabe, Specific heat measurement of the spinel compound $\mathrm{CuV}_{2} \mathrm{~S}_{4}$, AIP Conference Proceedings No. 850 (AIP, New York, 2006), p. 1317.

[26] S. Kawaguchi, H. Ishibashi, N. Tsuji, J. Kim, K. Kato, M. Takata, and Y. Kubota, Structural properties in incommensurately modulated spinel compound $\mathrm{CuV}_{2} \mathrm{~S}_{4}$, J. Phys. Soc. Jpn. 82, 064603 (2013).

[27] J. Mahy, D. Colaitis, D. van Dyck, and S. Amelinckx, Electron diffraction evidence for domain structures in the lowtemperature incommensurate phase in $\mathrm{CuV}_{2} \mathrm{~S}_{4}$, J. Solid State Chem. 68, 320 (1987).

[28] R. Horny, S. Klimm, M. Klemm, S. Ebbinghaus, G. Eickerling, and S. Horn, Electronic transport and structural properties of electronically correlated $\mathrm{CuV}_{2} \mathrm{~S}_{4}$, J. Magn. Magn. Mater. 272276, E307 (2004).

[29] C. Mujica, G. Carvajal, J. Llanos, and O. Wittke, Redetermination of the crystal structure of copper(I) tetrathiovanadate (sulvanite), $\mathrm{Cu}_{3} \mathrm{VS}_{4}$, Z. Kristallogr. NCS 213, 12 (1998).

[30] V. Dyadkin, P. Pattison, V. Dmitriev, and D. Chernyshov, A new multipurpose diffractometer PILATUS@SNBL, J. Synch. Rad. 23, 825 (2016).

[31] P. van der Linden, H. Vitoux, R. Steinmann, B. Vallone, and C. Ardiccioni, An open flow helium cryostat for synchrotron X-ray diffraction experiments, J. Phys.: Conf. Ser. 425, 012015 (2013).
[32] See Supplemental Material at http://link.aps.org/supplemental/ 10.1103/PhysRevB.99.195140 for details on the diffraction experiments and values of the structural parameters at each temperature.

[33] International Tables for Crystallography Vol. A, 5th ed., edited by T. Hahn (Kluwer Academic, Dordrecht, 2002).

[34] H. T. Stokes, B. J. Campbell, and S. van Smaalen, Generation of $(3+d)$-dimensional superspace groups for describing the symmetry of modulated crystalline structures, Acta Crystallogr. A 67, 45 (2011).

[35] A. G. Jackson, Handbook of Crystallography (Springer-Verlag, New York, 1991).

[36] V. Petricek, M. Dusek, and L. Palatinus, Crystallographic computing system JANA2006: general features, Z. Kristallogr. 229, 345 (2014).

[37] Z. W. Lu, B. M. Klein, E. Z. Kurmaev, V. M. Cherkashenko, V. R. Galakhov, S. N. Shamin, Y. M. Yarmoshenko, V. A. Trofimova, S. Uhlenbrock, M. Neumann, T. Furubayashi, T. Hagino, and S. Nagata, Electronic structure of $\mathrm{CuV}_{2} \mathrm{~S}_{4}$, Phys. Rev. B 53, 9626 (1996).

[38] J. Matsuno, A. Fujimori, L. F. Mattheiss, R. Endoh, and S. Nagata, Photoemission and band-calculation studies of the charge-density wave in $\mathrm{CuV}_{2} \mathrm{~S}_{4}$, Phys. Rev. B 64, 115116 (2001).

[39] A. Wölfel, L. Li, S. Shimomura, H. Onodera, and S. van Smaalen, Commensurate charge-density wave with frustrated interchain coupling in $\mathrm{SmNiC}_{2}$, Phys. Rev. B 82, 054120 (2010). 